\title{
Commemoration of the centenary of the birth of Mario Milletti (1914-1959), co-founder of Acta Neurochirurgica
}

\author{
Sebastiano Paterniti
}

Received: 23 November 2014 / Accepted: 9 February 2015 / Published online: 1 March 2015

(C) Springer-Verlag Wien 2015

The year 2014 marks the centenary of the birth of Mario Milletti, an Italian neurosurgeon and a pioneer of modern neurosurgery in Italy. In 1950, together with the Austrian Wolfram Sorgo, he founded Acta Neurochirurgica. For this reason, I believe a short commemoration in this journal is appropriate.

Milletti (Fig. 1) was born in Pescia (Pistoia) on 29 April 1914, and he graduated with a degree in medicine from the University of Bologna. Early in his career, he devoted himself first to histology and embryology and then to pathologic anatomy (for several months in 1936 and in 1937, he attended the Institute of Pathology at the University of Leipzig under Werner Huech's guidance); he wrote various works, 32, on histopathological topics, among which those concerning experimental modifications of liver cells are of particular importance. For a few years, he devoted himself to general surgery and was an assistant at the Surgical Clinic of Bologna University, first under the guidance of Raffaele Paolucci and then under the leadership of Gherardo Forni; his scientific production in the field of general surgery consists of 15 papers.

His work in neurosurgery began in 1942, at the age of 28 years. In 1942-43, he attended the Neurosurgical Clinic of Berlin with Wilheilm Tonnis, then the Neurosurgical Clinic in Stockholm with Herbert Olivecrona. In 1946, he spent 9 months at the Neurosurgical Clinic of Manchester with Sir Geoffrey Jefferson. Under the guidance of these masters of neurosurgery, Milletti improved his knowledge of diagnostic arteriography (with Tonnis), ventriculography and pneumoencephalography (with Olivecrona) as well as

S. Paterniti $(\bowtie)$

University of Messina Medical School, Messina, Italy

e-mail: bapate47@virgilio.it the surgical techniques for various neurosurgical diseases; in particular, in Manchester with Jefferson he was especially interested in cerebral aneurysms. In 1949-50, he visited the major neurosurgical centers in the United States. From 1947, Milletti was head of the Institute of Neurosurgery "Cesare Cavina" at the Maggiore Hospital in Bologna. He was one of the nine founders of the Italian Society of Neurosurgery (Turin, 29 May 1948) and a member of a number of Italian and foreign scientific associations. In 1950, he organized the "Neurochirurgicum Symposium" in Bologna, the first international neurosurgery meeting ever held in Italy.

In November 1951 at the first Congress of the Italian Society of Neurosurgery, he was a speaker on the topic of

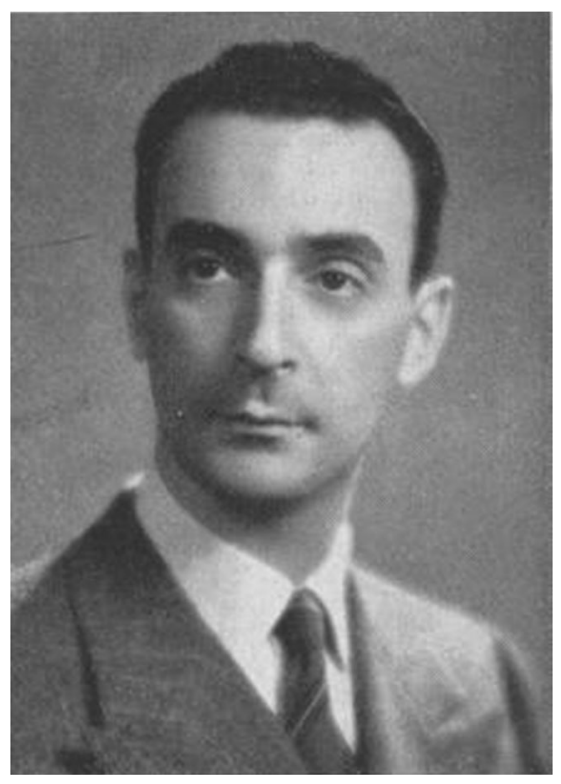

Fig. 1 Mario Milletti 
Fig. 2 Title page of Milletti's work, "Aneurysms of cerebral vessels. Clinical diagnosis and surgical treatment"
ISTITUTO NEUROCHIRURGICO . CESARE CAVINA - BOLOGNA

(Direttore: Prof. M. Mıs.rm)

\section{Gli aneurismi dei vasi cerebrali. Diagnostica clinica e terapia chirurgica $(")$.}

Prof, M. MiLlettit

\section{INTRODUZIONE}

Eे quasi certo che 25 anni or sono un lavoro sugli aneurismi dei vasi cerebrali avrebbe potuto essere fatto solo da un anatomopatologo è non da un clinico o da un neurochirurgo.

E questo infatti un capitolo che da non molto tempo ha lasciato le gelide, statiche sale dei settorati per passare alle vivaci, dinamiche discussioni della clinica.

Esattamente nel 1923 Srmonds e Cushisc sulla stessa rivista pubblicavano due lavori che dovevano essere in questo campo la prima pietra miliare: cinque anni più tardi l'introduzione dell'arteriografia cerebrale rappresentava la seconda pietra miliare nella storia degli aneurismi.

Questa tecnica provoco in un primo momento un grande entusiasmo e sembró oscurare parzialmente la osservazione clinica. Ma la diagnostica clinica prese di nuovo, rapidamente, il sopravvento, ed oggi l'arteriografia è chiamata in questo campo a confermare delle diagnosi, piuttosto che a risolvere dei problemi castali. In qualche paese d' Europa e d'America che ha più tardi degli altri introdotto l' uso della arteriografia, mi è capitato di sentir parlare di noi, che gia dell'arteriografia facevamo largo uso, come di "chasseurs d'images ». Puó darsi che nel campo degli aneurismi cerebrali noi siamo anche stati forse nei primi anni degli " chasseurs d'images " :

(") Una parte di questo materiale ì stato oggecto di una relazione al $1^{\text {" congress }}$ della Societa Kaliana di Neurochirurgia a Milano, Novembre 1951.

28 cerebral aneurysms. Milletti was very interested in saccular aneurysms, a subject on which he published several works $[7,9,10,14,15]$ outlining all aspects of aneurysms. Figure 2 shows the title page of a long article, 216 pages, entitled "Aneurysms of cerebral vessels. Clinical diagnosis and surgical treatment," published in 1952 [15], in which the author reports and expands on the lecture delivered the year before at the first Congress of the Italian Society of Neurosurgery. Some of the other papers reported cases presenting special features, such as multiple aneurysms $[3,4]$ or aneurysms of the optical-chiasmatic region [8]; Milletti reported cases he had observed at his Institute in Bologna and during his stays in Berlin, Stockholm and Manchester. 
Fig. 3 Title page of Acta

Neurochirurgica (1950), Suppl. 1

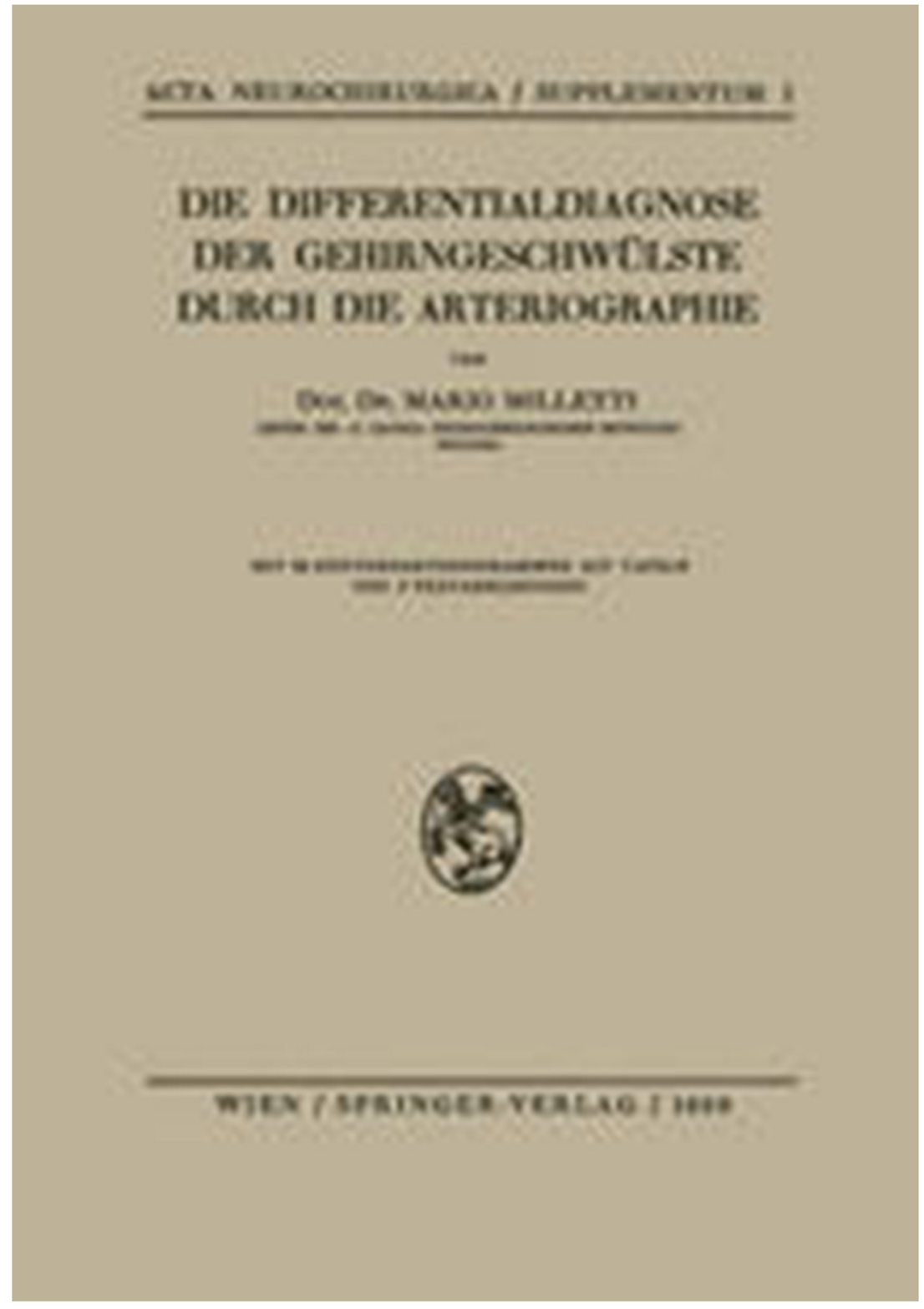

In the context of vascular diseases, two interesting works on thrombosis of the internal carotid artery in the neck [11] and of the posterior cerebral artery [18] should be pointed out. Milletti was interested in many other aspects of neurosurgical pathology, as is proved by his scientific works on brain tumors $[23,24]$, pediatric neurosurgery $[21,27,28,31]$, functional neurosurgery $[19,22,26,30]$ and other fields [16, 20, 29, 32].

Certainly he was very interested in diagnostic cerebral angiography, the subject of some of his publications $[5,6,17$, 25] and of a monograph [12]. His most important and best known work is probably a monograph, 79 pages long, published in 1951 in the first Supplement to Acta Neurochirurgica (Figs. 3 and 4a, b), dedicated to diagnostic arteriography of brain tumors [13]. In this, Milletti, based on 203 cases of cerebral tumors examined by means of angiography, investigated the possibility of establishing a differential diagnosis between different histological types, concluding that this is possible in an high percentage of glioblastomas and meningiomas.

Due to a serious illness, Mario Milletti died in Bologna in March 1959 at the age of 45 years. He was commemorated by Filippo Caramazza on 29 February 1960 at the Medical Society of Bologna [1] and also briefly mentioned by Tonnis in Acta Neurochirurgica [33]. In a speech celebrating the 25 th anniversary of the foundation of the Italian Society of Neurosurgery, Paolo Conforti, then president of the Society, said: "Mario Milletti and Marino Quarti Trevano (another founding member who died prematurely) are to all an example of the life, study, technique, and dedication to our discipline" [2]. 


\section{Erster Teil.}

\section{Einleitung.}

Es ist bekannt, daß die Tumoren des Zentralnervensystems, und insbesondere die Gehirntumoren, vergleicht man sie mit den in anderen Teilen des Organismus vorkommenden Tumoren, ein durchaus eigenes Verhalten erkennen lassen. Kranke mit Gehirntumoren liefern dem Chirurgen nur einen geringen Teil der klinischen Symptome, die ihn die Natur des Tumors, seine Gutartigkeit oder Bösartigkeit feststellen ließen. Dieser diagnostische Mangel Gutartigkeit oder Bösartigkeit feststellen ließen. Dieser diagnostische Mangel
muß sich in seiner ganzen Schwere auf die klinische Prognose und auf das muß sich in seiner ganzen Schwere auf die klinisch
auswirken, was der Operationsplan sein könnte.

Es war logisch, daß der Neurochirurg nach einem Mittel suchte, diese Liicke zu decken. Die Ventrikulographie war nicht imstande, hier mehr als eine nur beschränkte Unterstlitzung zu bieten. Aber die Gehirnangiographie, eine nur beschränkte Unterstuitzung zu bieten. Aber die Gehirnangiographie,
von der man sich im ersten Augenblick nur die Möglichkeit einer Diagnose von der man sich im ersten Augenblick nur die Möglichkeit einer Diagnose
auf Lokalisierung versprochen hatte, erwies bald ihre Eignung, Daten zu liefern, die über die Feststellung des Tumorsitzes hinaus für eine Diagnose auf dessen Natur nützlich sein konnten.

Diese von M o n i z erkannte Möglichkeit wurde in den folgenden Jahren von Moniz selbst, von seinem Schüler Almeida $\mathrm{L}$ ima und von Tönn is und seiner Schule Schritt für Schritt studiert. Die Schlußfolgerungen, zu denen die Neurochirurgen gelangt sind, stimmen heute ziemlich miteinander überein und gestatten die Möglichkeit einer Diagnose auf die Natur des Tumors im weiteren Sinn, nämlich ob es sich um einen gutartigen oder um einen bösartigen Tumor handelt. Aber hinsichtlich der Details, auf die eine solche Diagnose zu stützen ist, und vor allem hinsichtlich der Möglichkeit, an einer genaweren Diagnose vorzudringen, indem man die verchiedenen, sei es gut- oder bösartigen, Tumorformen radiographisch abscheidet, darüber geht die Meinung der Neurochirurgen auseinander.

\section{Ziel der Arbeit.}

Ziel dieser Arbeit ist es, das in den Jahren 1937 bis 1942 an der Neurochirurgischen Universitätsklinik zu Berlin gesammelte reiche Material unter dem Gesichtspunkt dieser Möglichkeit zu analysieren. Vor allem soll versucht werden, aus der minutiösen Prüfung der studierten Fälle, die in Tabellen einzeln beschrieben sind, jene radiographischen Zeichen herauszuarbeiten, die sich mit größerer Häufigkeit darstellen und daher eine größere Bedeutung in der Differentialdiagnose annehmen.

Die Möglichkeit, die Diagnose auf Bösartigkeit oder Gutartigkeit einer Neubildung mit einer gewissen Sicherheit stellen zu können, ist von gröBter

\section{Milletti, Die Differentialdiagnase der Gehimgeschwillste Durch die Arteriographit
OSpringer-Verlag in Vienna 1950}

Fig. 4 a, b First and second pages of Acta Neurochirurgica Suppl 1

\section{References}

1. Caramazza F (1960) Commemorazione del Socio Prof. Mario Milletti Bull Sci Med (Bologna) 132:526-534

2. Conforti P (1975) Discorso celebrativo del XXV Anniversario della Fondazione della Società Italiana di Neurochirurgia. J Neurosurg Sci 19:1-5

3. Milleti M (1946) Contributo alla conoscenza degli aneurismi multipli dei vasi cerebrali. Riv Oto-Neuro-Oftalm 21:141-153

4. Milletti M (1946) Su di un caso di doppio aneurisma dei vasi cerebrali. Bull Sci Med (Bologna) 21:181-183

5. Milletti M (1947) La diagnosi differenziale dei diversi tipi di tumori endocranici per mezzo dell'arteriografia cerebrale. Il Policlinico, Sez. Pratica 54:362

6. Milletti M (1947) La diagnosi arteriografica dell'ematoma subdurale. Mimerva Chir 2:66-68

7. Milletti M (1948) Diagnosi e classificazione degli aneurismi dei vasi cerebrali. Bull Sci Med (Bologna) 120:462-467

8. Milletti M (1948) La sindrome clinica degli aneurismi della regione ottico-chiasmatica. Riv Oto-Neuro-Oftalm 23:1-22

9. Milletti M (1949) Os aneurismas dos vasos cerebrais. Res Clin Cient 18:375-381

10. Milletti M (1949) Gli aneurismi dei vasi cerebrali. Rass Clin Sci 25: $48-54$

\section{Ziel der Arbeit.}

Bedeutung. Es gibt noch heute viele Chirurgen, die, beispielsweise, angesichts eines multiformen Glioblastoms von einem Eingriff absehen, während andere munis und interna

Uber die Feststellung der Charakteristika von Gutartigkeit oder Bösartigkeit eines Tumors hinaus ist es Absicht dieser Arbeit, zu untersuchen, ob e noch andere arteriographisehe Symptome gibt, durch die man zu einer genaueren und detaillierteren Diagnose über die Natur eines Tumors gelangen kann; im besonderen wurde die Möglichkeit geprüft, ob sich die arteriographische Diagnose auf folgende Formen von Gehirntumoren systematisieren lasse: multiformes Glioblastom, Meningeom, Astrocytom, Oligodendrogliom, Sarkom, Cholesteatom, Fernmetastasen im Gehirn.

\section{Allgemeine Bibliographie.}

M o n i z veröffentlichte schon 1927 in der „Revue neurologique“ einen ersten Fall, in dem die Diagnose auf Sitz des Tumors auf Grund der charak teristischen Eigengefäße gestellt wurde. Er stellt die Hypothese auf, es handle sich um ein Psammom (Angiosarkom). Trotzdem fehlt, da der Kranke nicht gestorben ist, die anatomische Bestätigung der radiologischen Diagnose.

Dieser ersten radiologischen Beobachtung von Meningeomen folgten seitens $\mathrm{M}$ o n i z Beschreibungen anderer Tumore, deren Diagnose ihre Natur betreffend auf Grund des Vorhandenseins eines eigenen Gefäßnetzes aufgestellt worden war. Diese Möglichkeit, auf die Natur von Gehirntumoren

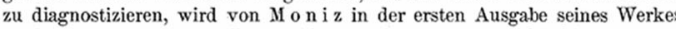
wieder erwähnt, das im Jahre 1931 erschienen ist; er bekräftigt sie noch weitgehender in der zweiten Auflage, veröffentlicht im Jahre 1934. In seinen Werken beschreibt der Verfasser einige typisch arteriographische Bilder, er schreibt (1931):

„Si la circulation des tumeurs est faite par un paquet d'artérioles minces que la radiographie dénonce, si on aperçoit des taches noires à l'endroit on se perd l'image des petites artères on peut diagnostiquer un méningoblastome." (S. 321.) Und weiter:

,La circulation est bien plus forte et l'endroit de la tumeur est indique par un réseau artériel très visible avec des artères assez grosses, mais sans qu'on puisse déterminer la tache de l'arrêt du liquide opaque dans la masse qu'on puisse déterminer la tache de l'arrêt du liquide opaque dans la
tumorale. Dans ces cas on doit penser aux astrocytomes, etc." (S. 321.)

Zum Schluß schreibt M on iz: "Cette différentiation a une certaine importance pour la prognostic et surtout pour l'intervention opératoire." (S. 321.)

Meningeome mit stark polimorpher histologischer Struktur, fibrilläre Gliome des ausgewachsenen Typus zeigen ein Zwischenbild zwischen den beiden oben beschriebenen. Zysten und Cholesteatome sind in ihrem Bild charakteristisch wegen des Fehlens von Gefäßen in der Tumorgegend: Die Diagnose auf Lokalisierung wird auf Grund der Ablenkung der Arterien gestellt.

11. Milletti M (1950) Does a clinical syndrome of primitive thrombose of the internal carotid artery at the neck exist? Acta Neurochir 1(2-3): $196-231$

12. Milletti M (1950) Angiografia Cerebrale. Ed. L. Parma, Bologna

13. Milletti M (1950) Die diffentialdiagnose der gehrngeschwulste durch die arteriographie. Acta Neurochir Suppl 1:1-79

14. Milletti M (1951) Contributo clinico alla patogenesi delle emorragie sub-aracnoidea spontanee. Le emorragie da rottura di aneurismi dei vasi cerebrali. Rass Clin Sci 27:15-19

15. Milletti M (1952) Gli aneurismi dei vasi cerebrali. Diagnostica clinica e terapia chirurgica. Arch Neurochir 1:433-649

16. Milletti M (1952) Results of neurosurgical treatment of opticochiasmatic arachnoiditis. Rev Oto-neuro-ophtalmol 24:294-305

17. Milletti M (1952) Arteriografia della vertebrale. Arch Neurochir 1: 301-330

18. Milletti M (1953) Angiographic demonstration of an isolated thrombosis of the Posterior Cerebral Artery. Acta Neurochir 3(4):301-305

19. Milletti M (1953) Results eloignes ( 4 ans $1 / 2-2$ ans) de 30 topectomies frontales. Rev Neurol (Paris) 88:448-550

20. Milletti M (1954) General anesthesia in neurosurgery. Zentralbl Neurochir 14:129-142

21. Milletti M (1955) Aspetti neurochirurgici dell'epilessia infantile. La Clin Pediatr (Bologna) 37:3-60

22. Milletti M (1956) A technique for continuous recording of brain potentials directly from the cortex: preliminary report. Acta Neurochir 4(4):470-476

23. Milletti M (1957) Principi generali di anatomia macro- e microscopica dei tumori dei nervi cranici. Relazione al VII 
Congresso della Società Italiana di Neurochirurgia (Miolano, 15-16 Dicembre 1956). Minerva Neurochir 1:29-41

24. Milletti M (1957) Possono la diagnosi e il trattamento dei tumori cerebrali essere migliorati? Primi orientamenti sull'uso degli isotopi radioattivi in Neurochirurgia. Bull Sci Med (Bologna) 129:245-248

25. Milletti M (1957) Risultati dell'uso dell'urografin nella arteriografia cerebrale. Radiol Med 43:737-749

26. Milletti M (1958) Introduzione agli interventi di stereotassi: considerazioni su un primo gruppo di 15 interventi di coagulazione del pallidus per morbo di Parkinson. Tip. L. Parma, Bologna

27. Milletti M (1959) Spina bifida e altre lesioni congenite del rachide e del midollo spinale. Ed. Scientifiche Istituto Cavina, Bologna
28. Milletti M, Martoni M (1956) Su di un caso di disembrioma cistico intrarachideo suppurato. La Clin Pediatr (Bologna) 38:85-96

29. Milletti M, Rizzi R (1947) Il quadro clinico dell'aneurisma arterovenoso del seno cavernoso con una osservazione personale. La Clin 11:114-130

30. Milletti M, Rovetta P (1957) The possible focal cortical genesis of some types of myoclonic epilepsy disclosed by performing a prefrontal lobotomy. Arch Neurobiol (Madrid) 4:171-194

31. Milletti M, Salvioli G (1953) L'ascesso cerebrale cosiddetto "paradosso" nei portatori di affezioni congenite del cuore. La Clin Pediatr (Bologna) 35:855-884

32. Milletti M, Suriani U (1953) Controlled hypotension with ganglioplegics in neurosurgery. Acta Neurochir 3(4):275-300

33. Tonnis W (1960) Mario Milletti gestorben. Acta Neurochir 8(1):103 